dass sie nur noch die rechtliche Grundordnung des Staates ist" (388).

An verschiedenen Stellen des Sammelbandes scheint also doch die ,neue Grundsätzlichkeit“ (379) der verfassungstheoretischen Diskussion durch, die Grimm beobachtet. Insofern vermag der Band das eingangs gemachte Versprechen einer Selbstvergewisserung des nationalen Staats- und Verfassungsrechts zumindest teilweise einzulösen.

Verena Frick

Fischer-Lescano, Andreas, Joachim Perels und Thilo Scholle (Hrsg.). Der Staat der Klassengesellschaft. Rechtsund Sozialstaatlichkeit bei Wolfgang Abendroth. Baden-Baden. Nomos 2012. 275 Seiten. $29,00 €$.

Die knappe Einleitung thematisiert die Rekonstruktion der „Eckpunkte der Abendrothschen Rechtstheorie" und die Suche „nach aktuellen Anschlussmöglichkeiten “ (10). Es folgen 13 Beiträge zur Biographie, zum Rechts- und Gesellschaftsverständnis und zum Demokratiekonzept. Eine Zusammenfassung fehlt. Frank Deppe, Peter Römer und Uli Schöler veranschaulichen mit ihren Unterschieden (61, 95 ff., 161 f., 168 f.) das Spektrum um „Abendroths Demokratieverständnis“. Allgemein geht es um die Geschichtlichkeit des Zusammenhangs von modernem Staat und bürgerlich-kapitalistischer Gesellschaft (so schon Kammler in der von Abendroth herausgegebenen Marburger „Einführung in die politische Wissenschaft“, 1968). Dies und direkte politische Konsequenzen machte Abendroth, wie es pathetisch heißt, für "Generationen kritischer Intellektueller" (61) anregend. Dies wäre Stoff einer
Retrospektive, die sich theorievergleichend verbindet mit Fragen nach der aktuellen Wirkung und Anregung. Abendroths Arbeiten und sein praktisches Engagement bilden immerhin einen Schlüssel für Aufstieg, Ausdifferenzierung und Erosion der Protestbewegungen um 1968 und 1970.

Wolfgang Abendroth (1906 - 1985) war von 1950 bis 1972 Professor für wissenschaftliche Politik an der Philosophischen Fakultät der Universität Marburg. Er war zunächst ein unangepasster, für den Verfassungs- und Sozialstaat, gegen die Notstandsgesetze votierender (149), mit studentischem Protest sympathisierender Marxist, der aus bevorzugt historischer Sicht Machtkonstellationen im antagonistischen Feld von Gesellschaft und Demokratie betrachtete. Ab 1970 entwickelt er, wie es beschönigend heißt, einen positiven „Bezug auf die Entwicklungen in der DDR" (11), er wird zum traditionalistischen Außenseiter (168), der 1973 mit einer privaten Karte gegenüber A. Norden Ulbrichts Tod bedauert. Schon davor vertritt Abendroth ein Telos, das Stalins Terror als ,unvermeidlich“ ansieht („Die Wende des Stalinismus“, 1956 - vgl. 161). Allgemein gilt: Negative Seiten müssen historisch in Kauf genommen werden (49). Abendroth hat ein kategoriales, Ausdifferenzierungen fremdes, methodisch geronnenes Verständnis von Marx, er beharrt auf Begriffen wie „Klassengesellschaft“ oder „Klassenkampf"; das Grundgesetz gilt ihm 1977 als „Klassenwaffenstillstand“ im „Kampf um Verfassungspositionen“ (Seifert 1966). Dabei betont Abendroth die „Einheit der Arbeiterklasse“ und rät in den 1970er Jahren „mindestens einmal in der Woche" zur Lektüre Lenins gegen „Linksradikalismus“ 
(162). Dies zeigt den Sprung in eine, hermetisch werdende, vom Fach und der Umwelt sich entfremdende Bezugswelt; getragen wird sie - was Abendroth widerspricht - von „Mosaik-Linken" vor allem in Gewerkschaften und Bildungseinrichtungen (194 ff.). Der Sammelband spricht von „Geradlinigkeit“, Abendroths Wandel an „radikaldemokratisch-sozialistischen Positionen“ drücke die „Widersprüche“ gegenwärtiger Politik aus (38). Bis auf eine Abwägung der Europapolitik (226 ff., 233 ff.) werben die Beiträge für Abendroth mit den Attributen „Völkerrechtler und Staatsrechtler, Politikwissenschaftler und Soziologe, Arbeitsrechtler und Verfassungsjurist, Historiker der Arbeiterbewegung und anderer sozialer Bewegungen, wissenschaftlicher Theoretiker wie tagespolitisch eingreifender Publizist“ (61). Als anschlussfähig an diese Kompetenzen werden Bereiche präsentiert, die Abendroth selbst nicht behandelt hat, wie Feminismus (117) und Weltgesellschaft (272).

Ein Widerspruch zwischen Abendroths „Lebensleistung“ und seinem späteren Verzicht auf Kritik selbst am „Russland des extremsten Stalinismus“ (Marburger Blätter 133, II/1970, 6, dagegen 161) wird angesprochen, nicht aber kritisch dargestellt. 1970 wird die „Denkmethode Lenins“ betont, ohne die "keine sinnvolle Politik“ zu führen sei, 1966 bezieht Abendroth sich auf "die Theorie des sozialistischen und demokratischen Humanismus“, 1968 begründet die Marburger Einführung in die Politikwissenschaft ihren Gegenstand und ihre westlich-marxistische Sicht mit Horkheimer (Kammler): Diese Entwicklungen mit ihren Konnotationen (Organisation versus Bewegung, Telos versus Unübersichtlichkeit) dürfte einen Schlüssel zum Verständnis der Frühphasen von Protest in der Bundesrepublik abgeben. Hans-Manfred Bocks Intellektuellengeschichte Abendroths (2001) und sein Vergleich der Frankfurter und Marburger „Schulen " verweisen darauf. Im Sammelband verschwimmt dies hinter vielen Einzelheiten. (Schöler [61] kündigt einen „eingehenden“ Beitrag an, über ein früheres Werk zum Thema hinaus. Zur Orthodoxie äußert sich Deppe [81 ff.]: Abendroth lebe einzigartig die Verbindung von Wissenschaft und Arbeiterbewegung [95], ohne „Klassenreduktionismus" [86] zu betreiben.)

Abendroth habe nichts an „Dringlichkeit" verloren (256), vor allem strategisch, für die "demokratische Frage“ liefere er "auch heute noch wichtige Hinweise“ (80, vgl. 97, 169, 196, 214): Es bleibt bei Wertungen. Der Band verzichtet auf einen Vergleich Abendroths im Kontext der sich als Fach konstituierenden Politikwissenschaft, so wie in den Werken von Bleek und Lietzmann 1999 (Bleek 2001 oder Balzer, Bock und Schöler 2001). Auch wird die Klassenanalyse nicht mit Theorien im Feld von Postmaterialismus, unkonventioneller Politik und Partizipation in Verbindung gebracht. Bereits vorliegende Darstellungen Abendroths als einen wissenschaftlichen Politiker, wie bei Balzer, Bock und Schöler 2001, mit „Aktualität", wie es bei Urban, Buckmiller und Deppe 2006 heißt, werden nochmals aufgegriffen, nicht aber weitergeführt. Vor allem wird die aktuelle Frage verschenkt, ob Abendroth helfen kann, eine neuerlich politisch-ökonomisch begründete politische Soziologie zu verstehen. Der Sammelband belässt es beim Hinweis, „dass auch die aktuellen Debatten durch einen Anschluss an Abendroths Verfassungs-, Rechts- 
und Demokratieverständnis bereichert werden können“ (12).

\section{Eike Hennig}

\section{Voigt, Rüdiger. Staatskrise. Muss sich die Regierung ein anderes Volk wählen? Staatsdiskurse, Bd. 12. Stuttgart. Franz Steiner Verlag 2010. 206 Seiten. 38,00€.}

Verfassung ist „in“, Staat ist „out“? Vielleicht. Aber wenn die Reihe „Staatsverständnisse“ mit mehr als fünfzig Bänden reüssiert, dann bieten Idee und Begriff des Staates offenbar noch reichlich Stoff für wissenschaftlichen Streit. Jedenfalls ist dem Reihengründer etwas gelungen, was nach den zahlreichen Abgesängen keineswegs selbstverständlich erschien, nämlich einen neuen Chor zu orchestrieren, in dem die moderne Staatlichkeit, ihre Herkunft und Zukunft, bei allen Varianten und Variationen das Hauptthema bildet. Diese Vielstimmigkeit bietet die Chance, eine weitere Reihe etablieren zu können, sinnigerweise „Staatsdiskurse" genannt - und auch hier greift der spiritus rector, nicht zum ersten Mal, selbst zur Feder. Im vorliegenden Fall, dem zwölften Band, aus drängendem Anlass.

„Muss sich die Regierung ein anderes Volk wählen?" Ein auf den ersten Blick fragwürdiger Untertitel. Denn bald schon macht die Lektüre deutlich: Rüdiger Voigt ist weder nach theatralischen Possen zumute, noch hat er Zeit für langatmige Lehrstücke in Brechtscher Manier. Das stellt bereits der Haupttitel klar: Staatskrise - Punkt. Auch die Essays haben etwas von diesem Stakkato; sie bestehen zumeist aus knappen Abschnitten, die kaum länger als einen Gedanke währen, und zwar auch bei den großen Themen. Ein Bei- spiel pars pro toto: „Weltfrieden, internationale Sicherheit und Gerechtigkeit“" (177), so der Titel eines Gliederungspunktes, der eine gewaltige Inhaltsfülle auf den Plan ruft, aber doch nur eine halbe Seite dauert. Wem das zu hastig ist, der wird keine Freude an dieser Kompilation haben. Denn um eine solche handelt es sich: Es ist eine Ausbeute von Notizen und Exzerpten, bits and pieces, die der Verfasser gesammelt und summiert hat und aus denen er das Porträt einer politischen Krisenzeit zusammenfügt, die seines Erachtens der Aufklärung bedarf.

Sechs der acht Essays (zählt man die Einleitung mit, die sich nicht in Komposition und Gehalt unterscheidet) haben sich diesem aufklärerischen Ziel ganz unmittelbar verschrieben. Die anderen beiden Beiträge, ebenfalls in kleinteiliger Art verfasst, richten sich gleichwohl aufs Grundsätzliche und haben stärker politiktheoretischen Charakter: Der Beitrag „Staat und Verfassung " (121-140) dreht sich um die Aktualität der Verfassungslehre Carl Schmitts, jener über „Konträre Staatsbilder" (143-159) handelt von den Leitmetaphern der Staatsphilosophie des Thomas Hobbes. Zunächst aber zur Krisendiagnose: Die Staatskrise, so wird man Voigt verstehen dürfen, verweist auf ein ganzes Bündel von Krisen, die miteinander verwoben sind. Grundlegend ist die „Legitimationskrise" des politischen Prozesses demokratischer Staaten, die wiederholt Thema der einzelnen Beiträge ist (24 ff., 55 ff.). Holzschnittartig zusammengefasst, liegt ihr wesentlicher Grund in der Entkopplung zwischen Politik und Volk. Die Folge davon? Es wächst sich eine politische Klasse aus, stumpf gegenüber den Anliegen und Bedürfnissen der Bürger, dafür umso anfälliger 